\title{
Dermatosis por Neblina Ácida. A propósito de un caso.
}

Patricio Amaro B', Mirentxu Alberdi $S^{2}$, Felipe Amaro $S^{3}$, Macarena Ahumada $B^{4}$.

'Universidad de Chile, Unidad de Dermatología Hospital Mutual de Seguridad; ${ }^{2}$ Médico Cirujano, Universidad de los Andes; ${ }^{3}$ Estudiante de Medicina, Universidad Mayor; ${ }^{4}$ Estudiante de Medicina $4^{\circ}$ año, Universidad de Chile.

\section{Resumen}

Las dermatosis laborales son patologías frecuentes en la práctica clínica y producen un problema importante en la salud de los pacientes, siendo la dermatitis de contacto ocupacional la más frecuente.

A continuación presentamos el caso clínico de un trabajador de la minería expuesto en su ambiente laboral a un aerosol ácido, llamado neblina ácida, presentando una hipopigmentación post inflamatoria secundaria a la exposición a éste. Tanto el proceso diagnóstico, como la prevención de las dermatitis de contacto laboral, debe ser un proceso riguroso, ya que su pronóstico es variable, incluso cuando se logre evitar la exposición al agente causal.

Palabras claves: neblina ácida, enfermedad laboral, hipopigmentación postinflamatoria.

\section{Summary}

Work-related dermatoses are frequent pathologies in the clinical practice and produce a major health-problem, being the occupational contact dermatitis the most frequent disease.

We study the case of a mining worker exposed in his work enviroment to an acid aerosol, called acid mist, presenting a post inflammatory hypopigmentation after the exposure to this acid. The diagnosis process, as well as the prevention of occupational contact dermatitis must be rigorous, since their prognosis is variable, despite of avoiding the exposure to the causative agent.

Key words: acid mist, laboral disease, post inflammatory hypopigmentation.

\section{Introducción}

Las dermatosis laborales representan una importante causa de discapacidad en el trabajo, con un considerable impacto en el trabajador, que puede conducir a un cambio de actividad laboral, interferencia en sus actividades sociales e incluso discapacidad permanente ${ }^{1-3}$. Dentro de las dermatosis laborales, la dermatitis de contacto (DC) son el tipo más frecuente, siendo la dermatitis de contacto irritativa $(\mathrm{DCl})$ y la dermatitis de contacto alérgica (DCA) aproximadamente un $80 \%$ y $20 \%$ de las dermatitis de contacto ocupacional (DCO) respectivamente ${ }^{4}$.

Las DCO, tanto alérgica como irritativa, pueden desarrollarse en cualquier etapa de la carrera profesional y son clínicamente indistinguibles de aquellas producidas por factores no ocupacionales. La única diferencia se basa en su causa. Es por ello que el proceso de evaluación de un paciente con DCO debe ser muy riguroso, precisando detalladamente en la historia clínica los síntomas, las sustancias que están en contacto con la piel durante la actividad laboral, tiempo de exposición, así como la mejoría de los síntomas al suspender la actividad y el empeoramiento al retomarla ${ }^{5}$.

La DCl ocupacional es una dermatitis iniciada y mantenida por la exposición temporal a agentes físicos o químicos ${ }^{6}$.Dentro de los agentes químicos conocidos, los metales tienen el potencial de causar DC irritativa y/o alérgica ${ }^{7}$. Los reportes de contactantes volátiles capaces de producir DC son relacionados, en la mayoría de los casos, a lugares de trabajo y, por lo general, los reportes son subestimados debido a la omisión de datos de contacto con polvos 0 agentes volátiles ${ }^{8}$.

\section{Caso Clínico}

Paciente de sexo masculino, 47 años de edad, con antecedentes de trombosis venosa profunda de pierna y trombo- 

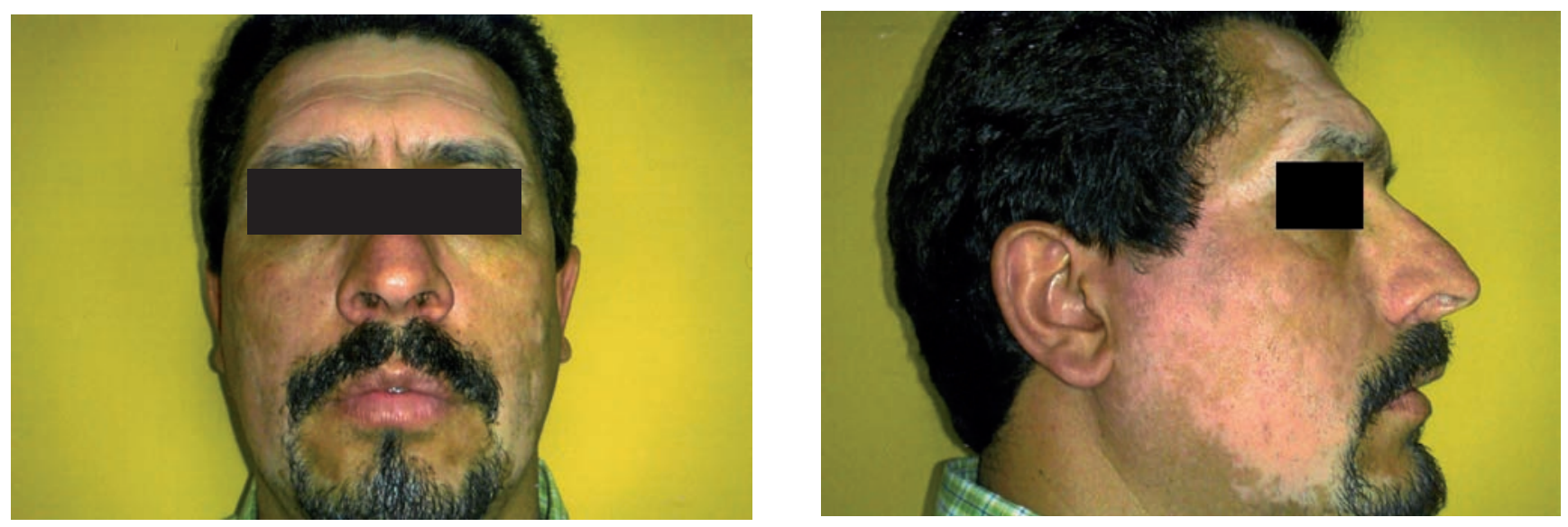

Figura 1 y 2. Se observa hipopigmentación simétrica en áreas fotoexpuestas

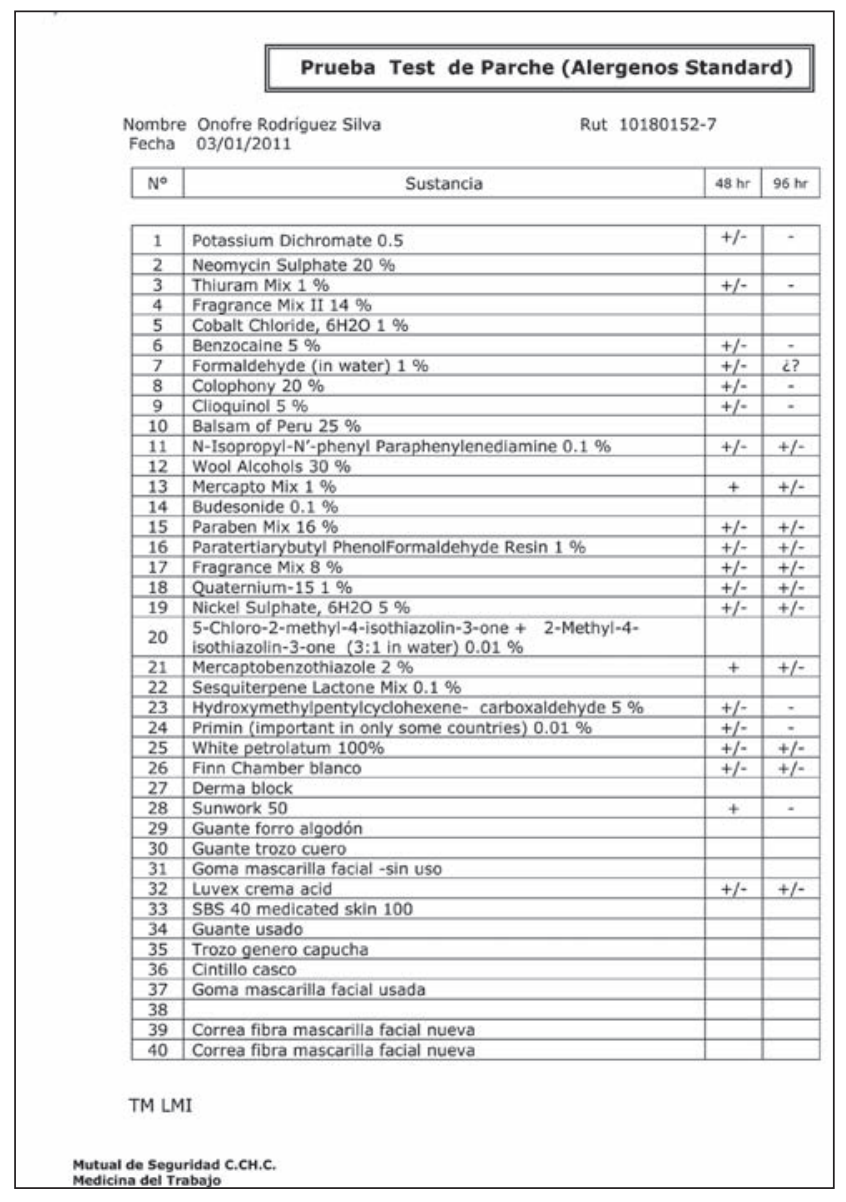

Figura 3. Prueba de parche estándar y laboral con reactividad en línea de fragancias. embolismo pulmonar en tratamiento con acenocumarol. Sin antecedentes personales ni familiares de vitiligo. Trabaja como operador de grúa y operario en área de electrolisis en la minería del cobre. Utiliza un dispositivo de protección facial (máscara) como elemento de seguridad. Consulta por cuadro de ocho años de evolución caracterizado los primeros cinco años por dermatitis facial, apareciendo en los últimos 3 años una hipopigmentación facial progresiva principalmente en la frente. Al examen físico presenta máculas hipopigmentadas confluentes en frente, mejillas y maxilares inferiores, coincidiendo con áreas en que los dispositivos de protección dejan descubiertas (Figuras 1 y 2). No presenta poliosis ni compromiso de otros sitios anatómicos. El tipo de lesión, ubicación y condiciones laborales lo hacen sugerente de ser secuela de contactantes a elementos de protección y ambiente. Se decide completar estudio con evaluación del puesto de trabajo (EPT) y prueba de parche estándar y laboral.

En la EPT se demostró que el paciente estuvo expuesto durante los primeros 5 años del cuadro clínico a un aerosol ácido producto del proceso electroquímico en la obtención del cobre, coincidiendo con la etapa de dermatitis facial que refiere el paciente. Durante ese período se desempeñaba como operario de grúa dentro de la nave. En los últimos 3 años mantenía su actividad de operario de grúa pero fuera de la nave, apareciendo la hipopigmentación mencionada anteriormente. Se decide realizar prueba de parche estándar que destaca múltiples reactividades a la línea de frangancias. (Figura 3) La prueba de parche laboral demuestra reactividad al luvex-crema ácida.

Se realiza biopsia de piel junto con tinción Fontana Masson, la cual demuestra reducción del número de melanocitos, lo 
que sugiere una hipopigmentación post inflamatoria (HPI), descartándose de este modo vitiligo.

Se inicia tratamiento tópico con gel en base a enzimas antioxidantes y vitamina A tópica sin resultados. Posteriormente se realiza tratamiento despigmentante en las zonas de piel sana con hidroquinona al $2 \%$, obteniéndose una respuesta parcial.

\section{Discusión}

La $\mathrm{DCl}$ es una enfermedad multifactorial que resulta de la exposición de la piel a un irritante ${ }^{9}$. Es un fenómeno complejo que depende de factores endógenos y exógenos, como la genética, la naturaleza de los irritantes y el medioambiente. Los agentes irritantes causan una reacción no específica al contacto con la piel interrumpiendo su función de barrera, causando de este modo daño celular directo a la epidermis y liberación de mediadores proinflamatorios ${ }^{10}$.

En relación al caso clínico presentado, el primer paso a seguir es distinguir si las lesiones faciales que presenta el trabajador corresponden a DCO irritativa o alérgica y luego distinguir si la DC corresponde o no a una enfermedad laboral.

El proceso diagnóstico implica una historia clínica detallada, examen físico, evaluación del puesto de trabajo y test de parche. Dentro de las pruebas de parche estándar y laboral el principal objetivo es la posible identificación de un alérgeno responsable, con el propósito de evitar la exposición ambiental del trabajador a ese alérgeno determinado, logrando en algunas ocasiones la recuperación clínica, como también evitar nuevos casos de la enfermedad ${ }^{11}$.

En la evaluación del puesto de trabajo del paciente, se observó que durante el proceso de electrobtención del cobre, al cual el trabajador estuvo expuesto, se producían cantidades significativas de vapores altamente tóxicos debido a la liberación de burbujas ricas en electrolitos, que al estallar, generaban un aerosol ácido conocido como neblina ácida.

La neblina ácida en altas concentraciones tiene un efecto perjudicial tanto para la salud del operador como para el medio ambiente, por esta razón se encuentra regulada por la legislación medioambiental ${ }^{12}$. Por otra parte, la sola presencia de contaminantes laborales en el lugar de trabajo no implica necesariamente que exista un potencial significativo de exposición; el agente debe estar en contacto con el trabajador. En el caso de las sustancias químicas, tanto la forma líquida como vaporizada del agente debe estar en contacto con el organismo o ser absorbido por él, para producir un efecto nocivo en la salud ${ }^{13}$.
En el caso del trabajador, al comienzo del cuadro clínico presentó una $\mathrm{DCl}$ secundaria al contacto directo con la neblina ácida en la región facial, que luego evolucionó a una hipopigmentación post inflamatoria (HPI), caracterizada al examen físico por máculas hipocrómicas en zonas no protegidas de la región facial.

Existe una "tendencia individual cromática" definida como una variación individual en la respuesta cutánea frente a procesos de inflamación o trauma. Algunos individuos pueden reaccionar con la producción normal, aumento o disminución de la melanina. Esto está genéticamente determinado y se hereda en forma de un patrón autosómico dominante. En general las personas con mayor susceptibilidad a daño cutáneo tienden a desarrollar hipomelanosis ${ }^{14}$.

La HPI se define como una pérdida adquirida parcial o total de la pigmentación de la piel que ocurre posterior a una inflamación cutánea. Puede ocurrir en cualquier fototipo de piel, siendo más común en el caso de fototipos altos. Esta puede ser localizada o generalizada, con una disminución, pero no ausencia de pigmento, lo que lo diferencia histológicamente de un vitiligo ${ }^{15}$.

El tamaño y forma de las lesiones hipopigmentadas por lo general se correlacionan con la distribución original de la dermatosis inflamatoria, lo que concuerda con el caso presentado.

Las lesiones pueden simular un vitiligo, con despigmentación en zonas alejadas de los sitios de la exposición.

Como conclusión es posible plantear que nuestro paciente presentó inicialmente una DCIO y posteriormente desarrolló una HPI secundaria, la cual hemos denominado "dermatosis por neblina ácida".

Actualmente no hay datos publicados en la literatura sobre $\mathrm{DCl}$ secundaria a neblina ácida. El caso presentado nos permite identificar agentes irritantes nuevos gracias a un proceso riguroso en la evaluación de dermatosis laborales.

La prevención de enfermedades profesionales de la piel puede realizarse mediante la aplicación de la jerarquía normal de los controles, es decir, eliminación, la sustitución, controles de ingeniería, prácticas de trabajo seguras y, cuando esto no sea posible, el equipo de protección personal ${ }^{8}$.

La prevención de la DCO es esencial, dado que el pronóstico de ésta es variable y algunos pacientes desarrollan trastornos de la pigmentación que pueden ser permanentes, a pesar de evitar la exposición al agente causal ${ }^{7}$. 


\section{Referencias bibliográficas}

1. Burnett CA, Lushniak B, Mc Carthy W, Kaufman J. Occupational Dermatitis Causing Days Away From Work in U.S. Private Industry, 1993. Am J Ind Med.1998;34(6): 568-573.

2. Cherry N, Meyer JD, Adisesh A, Brooke R, Owen-Smith V, et al. Surveillance of Occupational Skin Disease: EPIDERM and OPRA. Br J Dermatol.2000;142(6):1128-1134.

3. Koch P. Occupational Contact Dermatitis. Recognition and Management. Am J Clin Dermatol. 2001;2 (6): 353-365.

4. Lushniak B. Occupational contact dermatitis. Dermatologic Therapy. 2004;17:272-277.

5. Chee-Ching S, Chung-Shen C. Frequency and Determinants of Occupational Contact Dermatitis in 2793 Consecutively-Investigated Patients. Contact Dermatitis.1998;38:230-231.

6. Friis UF, Menné T, Schwensen JF, Flyvholm MA, Bonde JP, et al. Occupational irritant contact dermatitis diagnosed by analysisof contact irritants and allergens in the work environment. Contact dermatitis.2014;71(6):364-370.

7. Devantier C, Ejner K. Allergic contact dermatitis from a condensate of boric acid, monoethanolamine and fatty acids in a metalworking fluid. Contact dermatitis 2003;49(1): 45-46

8. Santos R, Goossens A. An update on airborne contact dermatitis: 20012006. Contact Dermatitis. 2007;57(6):353-360.
9. Levin $\mathrm{C} \mathrm{Y}$, Maibach $\mathrm{H} \mathrm{I}$. Irritant contact dermatitis: is there an immunologic component? Int Immunopharmacol.2002;2:183-189.

10. Slodownik D, Lee A, Nixon R. Irritant contact dermatitis: a review. Australas J Dermatol.2008;49:10-11.

11. Nicholson PJ, Llewellyn D, English JS. Evidence-based guidelines for the prevention, identification and management of occupational contact dermatitis and urticaria. Contact Dermatitis. 2010;63(4):177-186.

12. Vargas C, Navarro P. Aspectos preliminares de la evaluación y efecto de aditivos surfactantes en el proceso de electro obtención de cobre. Revista del Instituto de Investigaciones de la Facultad de Geología, Minas, Metalurgia y Ciencias Geográfica.2010;13(26):85-90.

13. Zubieta IX, Brown G, Cohen R, Medina E. Cananea Copper Mine. An International Effort To Improve Hazardous Working Conditions In Mexico. Int J Occup Environ Health.2009;15(1):14-20.

14. Ruiz-Maldonado $\mathrm{R}$, Orozco-Covarrubias ML. Postinflammatory hypopigmentation and hyperpigmentation. Semin Cutan Med Surg. 1997;16(1):36-43.

15. Vachiramon V, Thadanipon K. Postinflammatory hypopigmentation. Clin Exp Dermatol.2011;36(7):708-714.

\section{Evaluación (Quiz). Educación Médica Continua.}

\section{Respuestas correctas:}

1. d

2. d

3. e

4. b

5. C
6. c

7. b

8. C

9. b

10. e 